
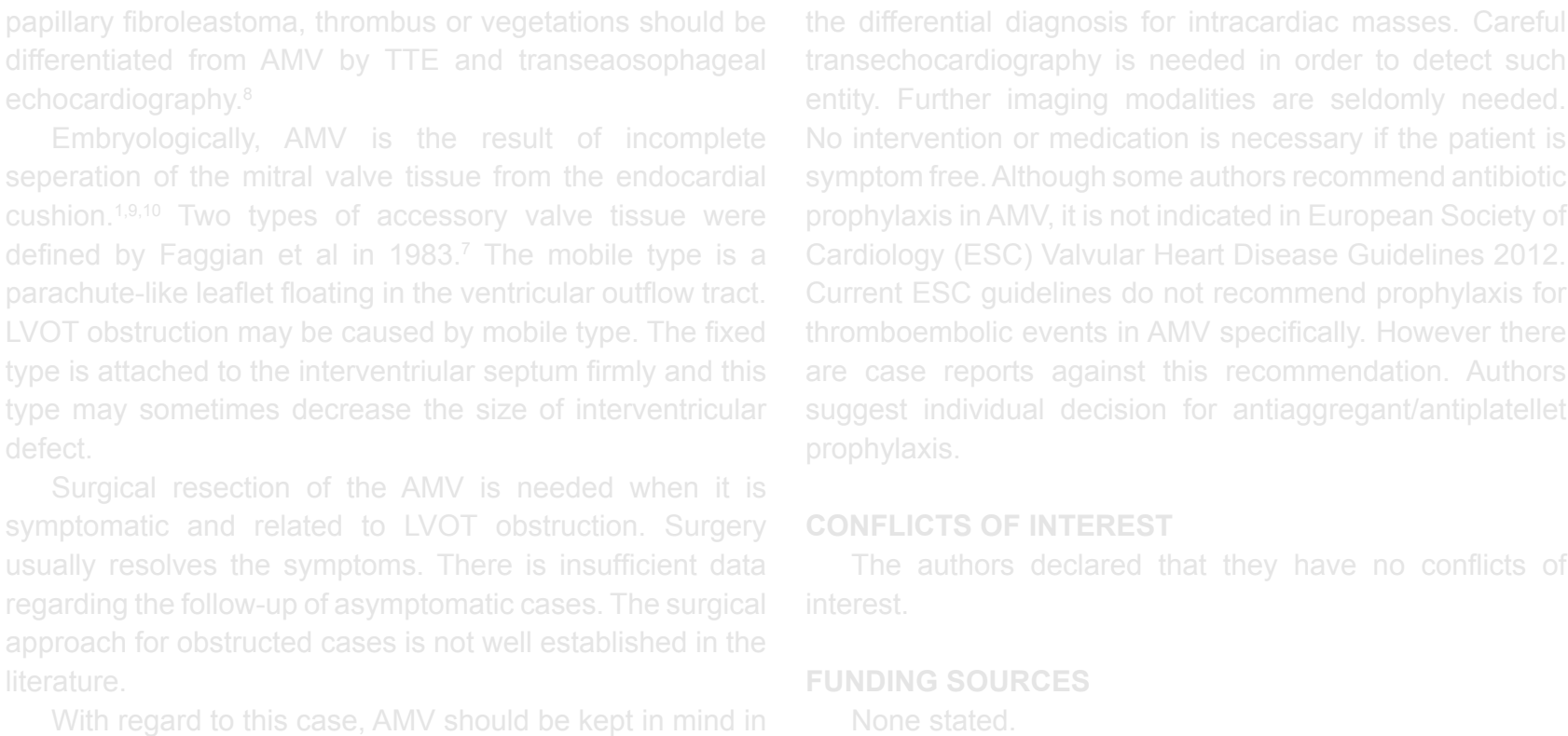

\title{
Parésia do Nervo Motor Ocular Comum em Contexto de Sinusite Esfenoidal
}

\author{
Third Cranial Nerve Palsy in Sphenoid Sinusitis
}

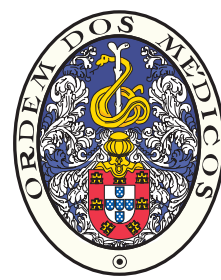

\author{
Luís Almeida DORES ${ }^{1}$, Marco Alveirinho SIMÃO¹, Marta Canas MARQUES ${ }^{1}$, Óscar DIAS 1 \\ Acta Med Port 2014 Nov-Dec;27(6):782-786
}

\section{RESUMO}

A patologia do seio esfenoidal é particular não só na sua apresentação clínica, mas também pelas suas complicações. Apesar de raras estas podem apresentar-se como défices dos pares cranianos, sendo importante ter um elevado índice de suspeição e estar familiarizado com o seu diagnóstico e abordagem terapêutica. Os sintomas são por vezes muito inespecíficos, contudo os mais frequentes são as cefaleias, alterações da acuidade visual e diplopia no contexto de disfunção de um ou mais nervos oculares motores. Reporta-se o caso de um indivíduo do sexo masculino, 59 anos de idade, que foi referenciado ao Serviço de Urgência Otorrinolaringologia por quadro de cefaleias frontotemporais de carácter progressivo com uma semana de evolução e agravamento clínico nas últimas 12 horas associando-se diplopia. O exame neurológico sumário não releva outras alterações para além da parésia do nervo motor ocular comum esquerdo. Na rinoscopia anterior/posterior não se verificava presença de massas ou rinorreia mucopurulenta. A Tomografia Computadorizada revelava preenchimento por componente tecidual de partes moles dos seios esfenoidais e erosão do

1. Departamento de Otorrinolaringologia, Voz e Perturbações da Comunicação. Hospital de Santa Maria. Faculdade de Medicina. Universidade de Lisboa. Lisboa. Port Recebido: 01 de Fevereiro de 2014 - Aceite: 09 de Abril de 2014 | Copyright @ Ordem dos Médicos 2014 
teto do seio esfenoidal esquerdo. Iniciou-se terapêutica endovenosa de largo espectro e corticoterapia sem melhoria clínica às 48 horas. Foi submetido a cirurgia endoscópica nassosinusal com boa resposta clínica e resolução completa da sintomatologia ao $10^{\circ}$ dia pós-operatório. Os autores apresentam este caso pela sua raridade e pela sua importância no diagnóstico diferencial de doentes com cefaleias e parésia de pares cranianos.

Palavras-chave: Sinusite Esfenoidal; Doenças do Nervo Oculomotor.

\section{ABSTRACT}

Sphenoid sinus disease is particular not only for its clinical presentation, as well as their complications. Although rare, these may present as cranial nerve deficits, so it is important to have a high index of suspicion and be familiar with its diagnosis and management. Symptoms are often nonspecific, but the most common are headache, changes in visual acuity and diplopia due to dysfunction of one or more ocular motor nerves. The authors report a case of a 59 years-old male, who was referred to the ENT emergency department with frontal headaches for one week which had progressively worsened and were associated, since the last 12 hours, with diplopia caused by left third cranial nerve palsy. Neurologic examination was normal aside from the left third cranial nerve palsy. Anterior and posterior rhinoscopy excluded the presence of nasal masses and purulent rhinorrhea. The CT scan revealed a soft tissue component and erosion of the roof of the left sphenoid sinus. Patient was admitted for intravenous antibiotics and steroids treatment without any benefit after 48 hours. He was submitted to endoscopic sinus surgery with resolution of the symptoms 10 days after surgery. The authors present this case for its rarity focusing on the importance of differential diagnosis in patients with headaches and cranial nerves palsies.

Keywords: Sphenoid Sinusitis; Oculomotor Nerve Diseases.

\section{INTRODUÇÃO}

A sinusite esfenoidal é uma entidade rara, clinicamente inespecífica e de difícil caracterização. Deste modo a sintomatologia mais frequente, cefaleias e alterações da visão, não permite definir um diagnóstico. Apesar da proximidade de estruturas importantes como a artéria carótida interna, o nervo óptico, a duramáter e os pares craneanos (III a VI), os sintomas relacionados com estas estruturas ocorrem muito raramente. ${ }^{1-3}$

É importante um elevado índice de suspeição pois a sinusite esfenoidal pode complicar originando défice permanente de pares cranianos, podendo até ser fatal, quando associada a complicação intracraniana. Lawson e Reino ${ }^{1}$ relatam que lesões inflamatórias nasossinusais do seio
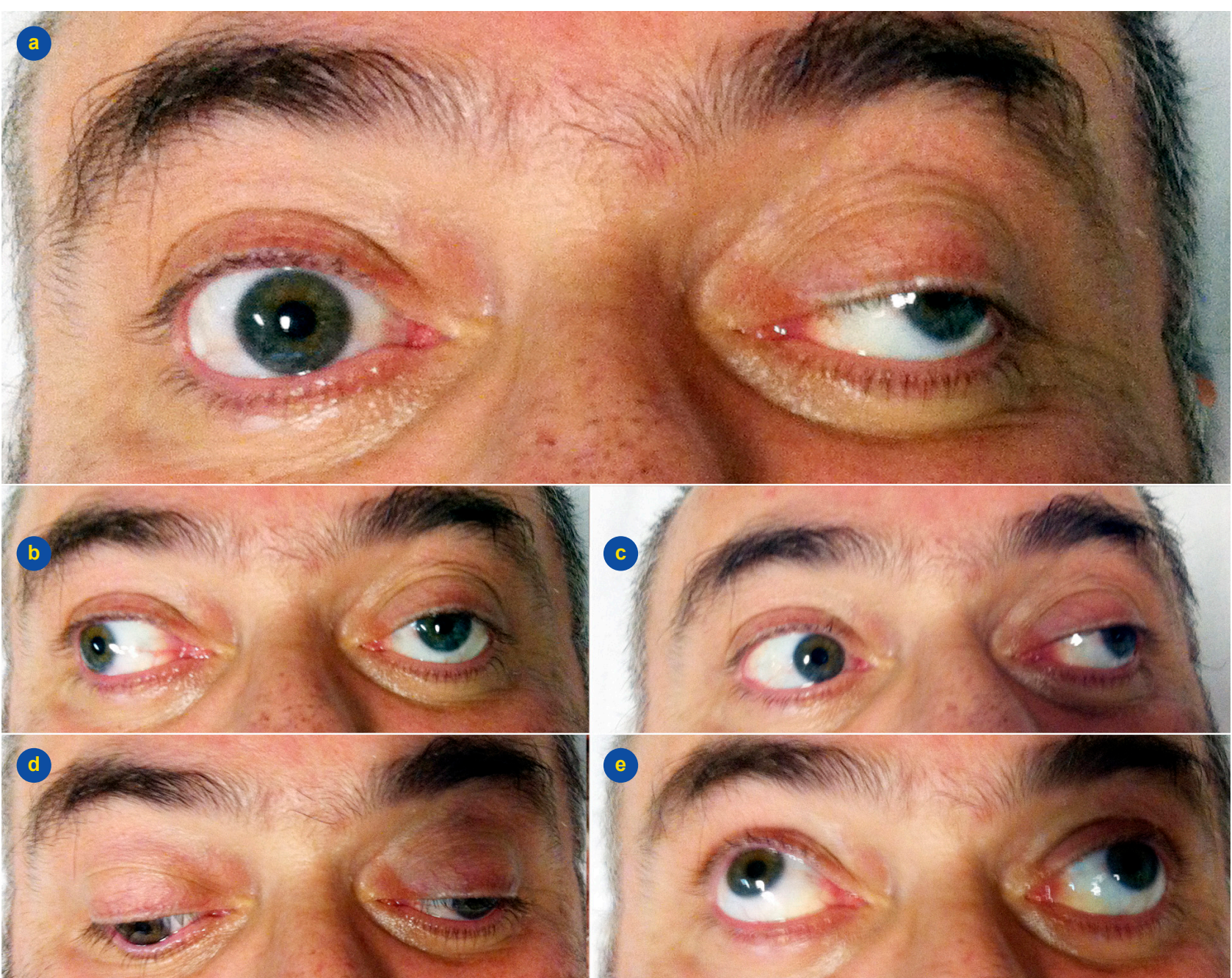

Figura 1 - Movimentos oculares na admissão. Alteração dos movimentos oculares do globo ocular esquerdo. Ptose da pálpebra à esquerda. $a$ - olhar em frente; $b$ - olhar para a direita; $c$ - olhar para a esquerda; $d$ - olhar para baixo; e - olhar para cima. 
esfenoidal complicam de diminuição da acuidade visual em $12 \%$ dos casos, ou de parésia de pares cranianos (III a VI) em $12 \%$ dos casos. O diagnóstico e tratamento baseiam-se na combinação da clínica, endoscopia nasal e exames de imagem: Tomografia Computadorizada (TC) associada, em casos específicos, a Ressonância Magnética (RM). É ainda discutível qual a fase adequada para a intervenção cirúrgica em caso de compromisso de pares cranianos. Estão descritos casos de recuperação total de parésia do nervo motor ocular externo (VI par) com 96 horas de evolução, após tratamento médico e cirúrgico. ${ }^{4,5}$

Em doentes com sinusite esfenoidal aguda a antibioterapia endovenosa é o tratamento de primeira linha. A abordagem cirúrgica do seio esfenoidal por via endoscópica deve ser realizada se, sob tratamento médico, a sintomatologia persistir ou agravar após 24 a 48 horas ou se ocorrerem sinais de complicações (alteração do estado de consciência, convulsões, aparecimento de sinais neurológi- $\cos$ focais)..$^{1-3}$

Os autores apresentam um caso clínico de uma complicação rara de sinusite esfenoidal, a parésia do motor ocular comum (III par). Com base neste caso fazem uma revisão dos aspectos clínicos e terapêuticos da patologia do seio esfenoidal, destacando a importância do diagnóstico diferencial em doentes com cefaleias e sintomas neurológicos.

\section{CASO CLÍNICO}

Os autores relatam o caso de um doente, sexo masculino, de 59 anos de idade, caucasiano. Como antecedentes pessoais apresentava: Diabetes mellitus tipo II e dislipidémia; vigiadas e controladas com metformina (1000 $\mathrm{mg} \mathrm{2x/} \mathrm{dia);} \mathrm{sinvastatina/ezetimibe} \mathrm{(20} \mathrm{mg/} 10 \mathrm{mg} \mathrm{1x/} \mathrm{dia);}$ hipertensão arterial medicada com losartan/hidroclorotiazida (50 mg/ 12,5 mg). Recorreu ao serviço de urgência por quadro clínico de cefaleias frontotemporais de predomínio esquerdo com sete dias de evolução. Ao sétimo dia refe-

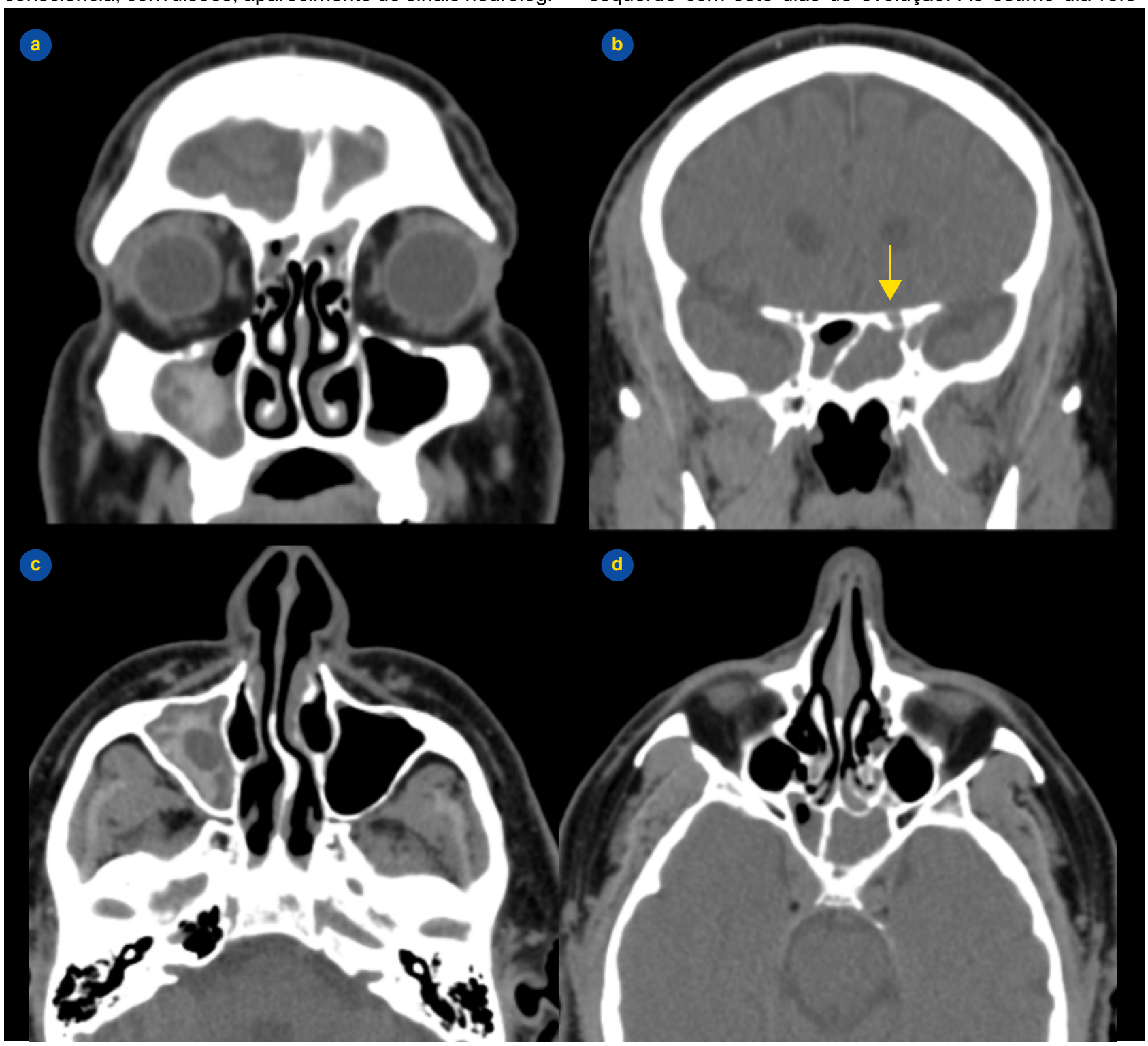

Figura 2 - Tomografia computadorizada revelando preenchimento bilateral por componente de tecidos moles de predomínio etmoidal e do seio esfenoidal (Fig.s 2a, 2d). Solução de continuidade do teto do seio esfenoidal esquerdo (Fig. 2b, seta). Conteúdo heterogéneo do seio maxilar esquerdo (Fig. 2c). 

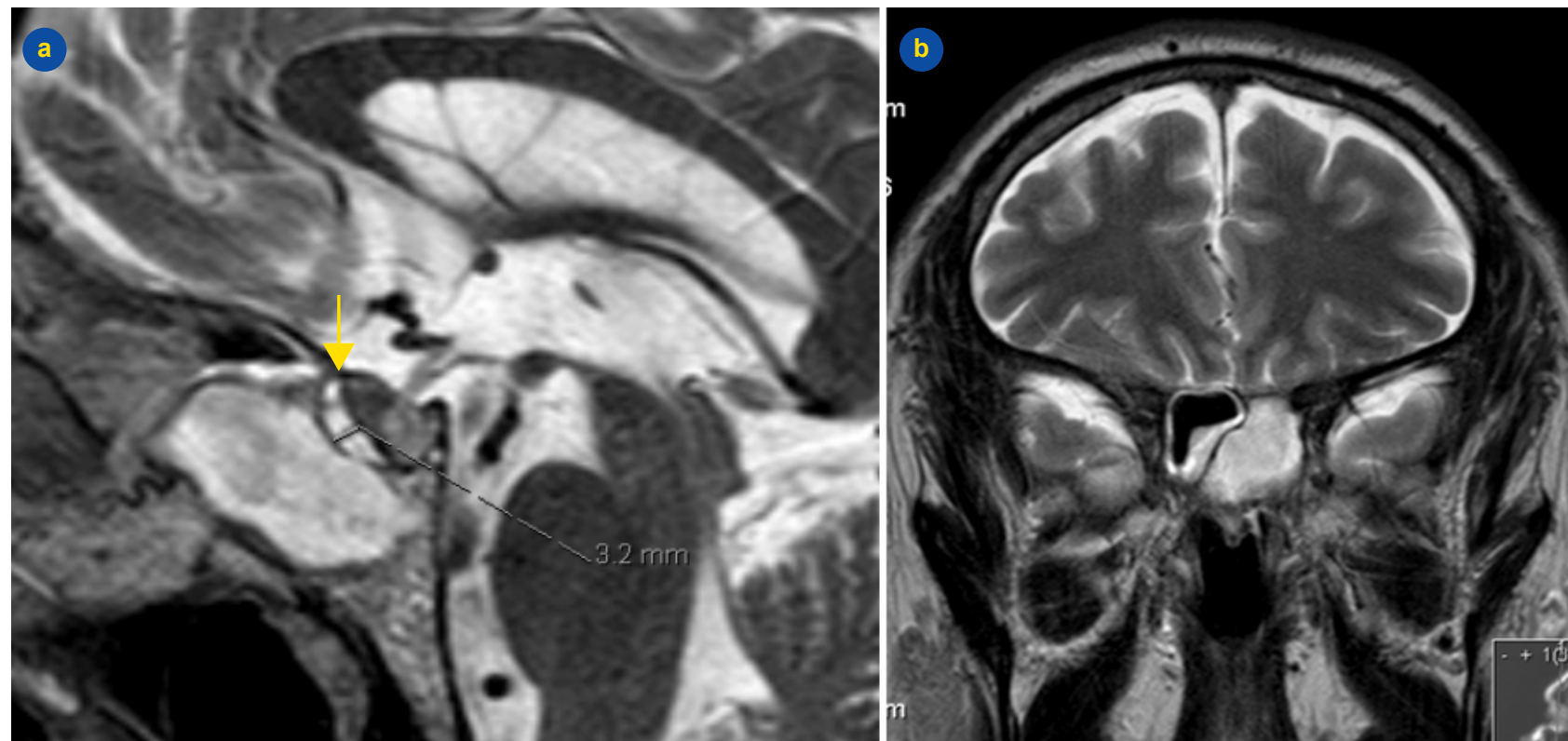

Figura 3 - Ressonância magnética revelando extensão intracraniana do processo inflamatório do seio esfenoidal esquerdo (Fig. 3a, seta) e preenchimento do seio esfenoidal esquerdo (Fig. 3b).

riu agravamento clínico com aparecimento de diplopia. Ao exame objetivo verificou-se alteração dos movimentos oculares com diplopia horizontal em supralevo, levo e infralevoversão (Fig. 1). Os reflexos pupilares estavam mantidos e simétricos, com acuidade visual e fundo ocular normal. $\mathrm{Na}$ rinoscopia anterior/posterior não se registaram alterações de relevo. Realizou TC cranioencefálica e dos seios perinasais que revelou preenchimento homogéneo por componente tecidual de partes moles dos recessos frontoetmoidais, etmóides e seios esfenoidais e preenchimento heterogéneo do seio maxilar direito (Fig. 2). Salientava-se discreta erosão da parede postero-superior do teto do seio esfenoidal esquerdo, em continuação com o pavimento da sela turca (Fig. 2b). Por suspeita de invasão intracraniana o doente realizou RM que confirmou extensão da componente inflamatória ao compartimento intracraniano, definindo-se lâmina hiperintensa (ponderação T2) com cerca de $3 \mathrm{~mm}$ de espessura que moldava a face anterior da hipófise e a desviava posteriormente (Fig. 3). Este achado foi interpretado como extensão inflamatória intracraniana, sem formação de colecção abcedada. Os seios cavernosos eram simétricos e permeáveis. $O$ doente não realizou exames com injecção de contraste por antecedentes alérgicos ao mesmo.

O doente foi internado e iniciou terapêutica endovenosa com ceftriaxona $2 \mathrm{gr}$ de 12/12 horas e prednisolona $50 \mathrm{mg} \mathrm{12/12} \mathrm{horas.} \mathrm{Após} 48$ horas sem melhoria clínica significativa, o doente foi submetido a cirurgia endoscópica nasossinusal com realização de esfenoidotomia esquerda por via endoscópica transetmoidal e etmoidectomia anterior parcial direita com sinusotomia maxilar tipo II, com saída de exsudado purulento. $O$ estudo microbacteriológico e micológico foi negativo assim como as biópsias da mucosa.

O doente apresentou melhoria clínica progressiva e resolução completa da parésia do III par ao $10^{\circ}$ dia de pós-operatório, encontrando-se assintomático desde há 12 meses.

\section{DISCUSSÃO}

As patologias do seio esfenoidal apresentam-se como um desafio clínico e terapêutico. As suas manifestações clínicas são semelhantes independentemente da sua etiologia. ${ }^{1,2}$ Cakman et al $^{2}$ reportam incidência de $72,5 \%$ de cefaleias em doentes com doença esfenoidal isolada. Do mesmo modo, Martin et $\mathrm{al}^{6}$ descrevem esta sintomatologia em $69 \%$ dos doentes. As alterações visuais são a segunda queixa mais frequente, com relatos de incidência de $21 \%{ }^{1,2,6}$ Apesar destas alterações serem mais frequentes na patologia neoplásica maligna, ocorrem também nos processos inflamatórios. Deste modo as alterações visuais não fornecem informação específica relativamente à natureza da lesão. Assim, destaca-se a necessidade da inclusão no diagnóstico diferencial de cefaleias associadas a alterações visuais, o diagnóstico de sinusite esfenoidal complicada. A presença isolada de cefaleias de predomínio occipital ou retro-orbitárias, deve alertar o clínico para o diagnóstico de sinusite esfenoidal isolada. ${ }^{7}$

As alterações visuais podem ser classificadas em dois grupos: diminuição da acuidade visual e diplopia (disfunção oculomotora). De acordo com Lee et al, ${ }^{8}$ o nervo óptico é o par craniano mais afectado (54\%), seguindo-se o nervo motor ocular comum (40\%). As afecções do nervo óptico incluem a neuropatia compressiva e a nevrite óptica sendo que ambas são consideradas emergências cirúrgicas que devem ser abordadas nas primeiras 72 horas de evolução. A resolução clínica é mais frequente no casos de disfunção oculomotora do que nos casos de diminuição da acuidade visual. A recuperação poderá ocorrer ao longo de vários meses no período pós-operatório sendo que são raras as recuperações em doentes com alterações visuais por períodos superiores a seis meses. ${ }^{5}$

A endoscopia nasal na patologia do seio esfenoidal permite uma avaliação da mucosa e anatomia das fossas nasais, sendo útil para obter material para cultura e definir 
se existe extensão para a cavidade nasal. A TC está indicada em todos os casos de sinusite esfenoidal complicada. Esta permite definir a extensão da lesão e a identificação de possíveis focos de erosão das paredes do seio esfenoidal. A RM está indicada quando a evolução clínica não é favorável, nos casos de erosão óssea ou quando são identificadas massas de componente de tecido mole na porção lateral e superior do seio esfenoidal. ${ }^{6}$

As abordagens cirúrgicas endonasais das lesões do seio esfenoidal incluem a endoscópica transnasal, a transetmoidal, a transseptal e a via da fossa pterigomaxilar. Estas técnicas podem ser associadas a abordagem por via externa quando necessário. Nas situações inflamatórias, a esfenoidotomia por via endoscópica transnasal usando o corneto superior como referência é uma técnica considerada eficaz e segura. ${ }^{9-11} \mathrm{Em}$ casos específicos poderá realizar-se uma recessão parcial do corneto médio para melhor visualização. A abordagem transetmoidal poderá ser considerada em fossas nasais pouco amplas ou nos casos de patologia concomitante de outros seios perinasais. Deve considerar-se esta abordagem quando existem complicações de sinusite esfenoidal e se se pretende um maior controlo e drenagem no período pós-operatório. A abordagem transseptal permite uma exposição alargada do seio esfenoidal e uma visão binocular do mesmo. É utilizada principalmente na cirurgia da hipófise podendo também ser adaptada à patologia do seio esfenoidal isolada.

\section{REFERÊNCIAS}

1. Lawson W, Reino AJ. Isolated sphenoid sinus disease: an analysis of 132 cases. Laryngoscope. 1997;107:1590-5.

2. Cakmak O, Shohet MR, Kern EB. Isolated sphenoid sinus lesions. Am J Rhinol. 2000;14:13-9.

3. Miller C, Suh JD, Henriquez OA, Schlosser RJ, Ramakrishnan VR, Chiu AG. Prognosis for sixth nerve palsy arising from paranasal sinus disease. Am J Rhinol Allergy. 2013;27:432-5.

4. Lee LA, Lee TJ, Huang CC. Endoscopic sinus surgery for solitary abducens palsy in patients with isolated sphenoid sinus disease: report of four cases. Chang Gung Med J. 2002;25:689-94.

5. Bizzoni A, Bolzoni Villaret A, Lombardi D, Tomenzoli D, Danzi P, Semeraro $F$, et al. Isolated sphenoid inflammatory diseases associated with visual impairment: 15-year experience at a single institution. Rhinology. 2011;49:202-6.

6. Martin TJ, Smith TL, Smith MM, Loehrl TA. Evaluation and surgical

\section{CONCLUSÃO}

Apesar de a cefaleia ser um sintoma muito inespecífico, pode estar associada a patologia do seio esfenoidal. A presença de cefaleias retro-orbitárias ou cefaleias associadas à presença de perturbações da visão, deve alertar o clínico para a pesquisa de doenças dos seios perinasais. É por isso necessário um elevado índice de suspeição, pois a intervenção precoce pode impedir a evolução da doença e o aparecimento de sequelas neurológicas permanentes.

\section{OBSERVAÇÕES}

Trabalho apresentado sob a forma de Poster no $24^{\text {th }}$ Congress of the European Rhinologic Society / $31^{\text {st }}$ International Symposium on Infection and Allergy of the Nose realizado em Toulouse (França), 17 a 21 de Junho, 2012.

\section{CONFLITOS DE INTERESSE}

Os autores declaram não ter quaisquer conflitos de interesse relativamente ao presente artigo.

\section{FONTES DE FINANCIAMENTO}

Não existiram quaisquer fontes externas de financiamento na elaboração do presente trabalho.

management of isolated sphenoid sinus disease. Arch Otolaryngol Head Neck Surg. 2002;128:1413-9.

7. Sieskiewicz A, Lyson T, Olszewska E, Chlabicz M, Buonamassa S, Rogowski M. Isolated sphenoid sinus pathologies - the problem of delayed diagnosis. Med Sci Monit. 2011;17:CR180-4.

8. Lee LI, Huang CC, Lee TJ. Prolonged visual disturbances secondary to isolated sphenoid sinus disease. Laryngoscope. 2004;114:986-90.

9. Friedman A, Batra PS, Fakhri S, Citardi MJ, Lanza DC. Isolated sphenoid sinus disease: etiology and management. Otolaryngol Head Neck Surg. 2005;133:544-50.

10. Loehrl TA, Smith TL. Computer-guided endoscopic sinus surgery. Curr Opin Otolaryngol Head Neck Surg. 2001;9:53-6.

11. Socher JA, Cassano M, Filheiro CA, Cassano P, Felippu A. Diagnosis and treatment of isolated sphenoid sinus disease: a review of 109 cases. Acta Otolaryngol. 2008;128:1004-10. 


\section{Parésia do Nervo Motor Ocular Comum em Contexto de Sinusite Esfenoidal}

Acta Med Port 2014:27:782-786

Publicado pela Acta Médica Portuguesa, a Revista Científica da Ordem dos Médicos

Av. Almirante Gago Coutinho, 151

1749-084 Lisboa, Portugal.

Tel: +351218428215

E-mail: submissao@actamedicaportuguesa.com

www.actamedicaportuguesa.com

ISSN:0870-399X | e-ISSN: 1646-0758

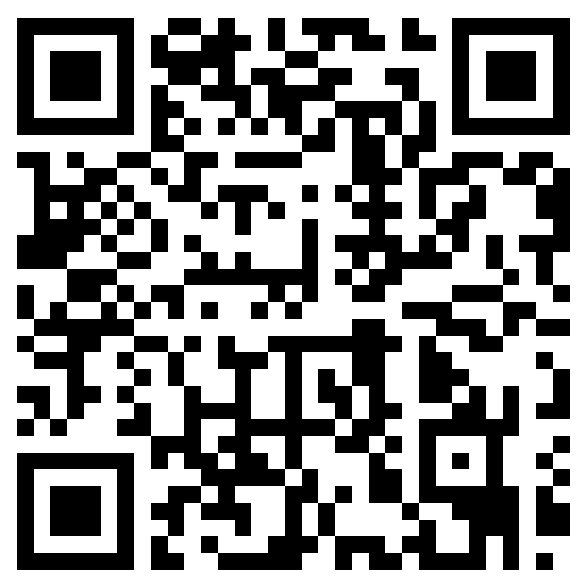

